\title{
Human papillomavirus DNA in glandular lesions of the uterine cervix
}

\author{
H Samaratunga, N Cox, R G Wright
}

\begin{abstract}
Aims-To assess the role of human papillomavirus in the pathogenesis of adenocarcinoma in situ, endocervical glandular dysplasia (a presumed precursor of adenocarcinoma) and endocervical glandular epithelial giant cell change.

Methods-Viral detection was carried out using an in situ hybridisation technique on paraffin wax sections. Biotinylated probes for human papillomavirus types $6 / 11,16 / 18,31 / 33 / 35$ were used with a colorimetric detection system.

Results-Nine out of $21(43 \%)$ cases of adenocarcinoma in situ contained human papillomavirus types $16 / 18$, one of which was also positive for $31 / 33 / 35$. Ten cases of glandular dysplasia and four cases of glandular epithelial multinucleation did not react with the probes used. Conclusions-These results indicate that while adenocarcinoma in situ is strongly associated with human papillomavirus infection, endocervical glandular dysplasia and glandular epithelial multinucleation are probably not associated with the virus.
\end{abstract}

(F Clin Pathol 1993;46:718-721)

Human papillomavirus (HPV) has been implicated in the pathogenesis of a variety of cervical epithelial lesions. A well known relation exists between virally induced nonneoplastic lesions (koilocytotic lesions) and cervical intraepithelial neoplasia (CIN) in cases of squamous epithelial lesions of the cervix..$^{1-3}$ It is also accepted that $\mathrm{CIN}$ is a precursor of invasive squamous cell carcinoma of the cervix. Certain types of HPV, such as HPV $16 / 18$ and $31 / 33$ are often associated with higher grade squamous epithelial dysplasias and with invasive squamous cell carcinomas. ${ }^{4}$ A cervical glandular epithelial lesion definitively induced by HPV, equivalent to koilocytosis, has not been recognised. The presence of bizarre multinucleate cells lining endocervical glands ${ }^{5}$ as well as glandular dysplasia have been suggested as being possibly induced by HPV. When endocervical glandular dysplasia is considered, reports are conflicting. By using sensitive ${ }^{3} \mathrm{H}$-labelled HPV 16 and HPV 18 probes, Tase et al found that only two out of 36 cases of glandular dysplasia contained HPV DNA. ${ }^{6}$ These two were lesions coexisting with adenocarcinoma in situ. It was concluded, therefore, that glandular dysplasia was unlikely to be a precursor lesion of adenocarcinoma and that a large proportion of glandular dysplasias were probably reactive lesions.

Jaworski used an in situ hybridisation technique with biotin labelled DNA probes for HPV on paraffin wax sections. ${ }^{7} \mathrm{He}$ demonstrated staining for HPV DNA in six out of 13 cases of endocervical glandular dysplasia (four cases showed the presence of HPV 18 DNA and two cases showed the presence of HPV 16 DNA).

Several studies have also shown that HPV DNA is present in endocervical adenocarcinoma in situ and invasive adenocarcinoma. ${ }^{8-10}$ The question of whether glandular dysplasia is a virally induced lesion is still largely unresolved. Similarly, the question of whether glandular dysplasia predisposes to adenocarcinoma is unanswered. Adenocarcinoma in situ, however, seems to be a precursor lesion of invasive adenocarcinoma. In one study it was shown that similar HPV types were found in both the adenocarcinoma in situ lesions and concomitant invasive adenocarcinoma lesions in all HPV positive cases. ${ }^{8}$

In an attempt to clarify whether HPV was present in the postulated precursor glandular lesions, HPV detection was carried out in cases of glandular dysplasia, glandular epithelial giant cell change, and adenocarcinoma in situ.

\section{Methods}

Twenty one cases of adenocarcinoma in situ of the uterine cervix obtained from the surgical pathology files of the Royal Brisbane Hospital were reviewed, together with 10 cases of endocervical glandular dysplasia and four cases in which there was multinucleation of endocervical glandular lining epithelium. Histological sections of cone or hysterectomy specimens of these cases were reviewed by two pathologists (HS and RGW). Histological diagnosis in each case was confirmed and the best representative section containing the glandular lesion in question was selected for each case. The corresponding formalin fixed, paraffin wax embedded tissue blocks were then retrieved for HPV testing. Six of the cases of adenocarcinoma in situ showed concomitant CIN. CIN, however, was present in the selected block in only four of the cases. In the other two cases CIN was present elsewhere in the cervix. 


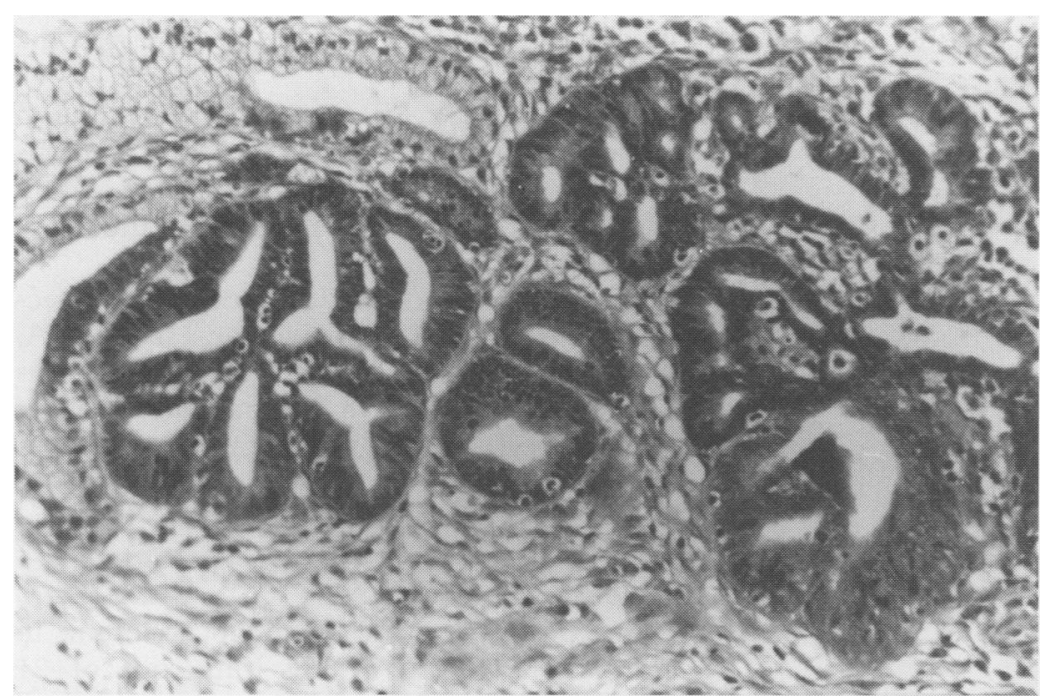

Figure 1 Adenocarcinoma in situ of the uterine cervix. Minor crypt architectural atypia with crypt budding is evident. A sharp transition is seen between normal and neoplastic epithelium in some affected glands.

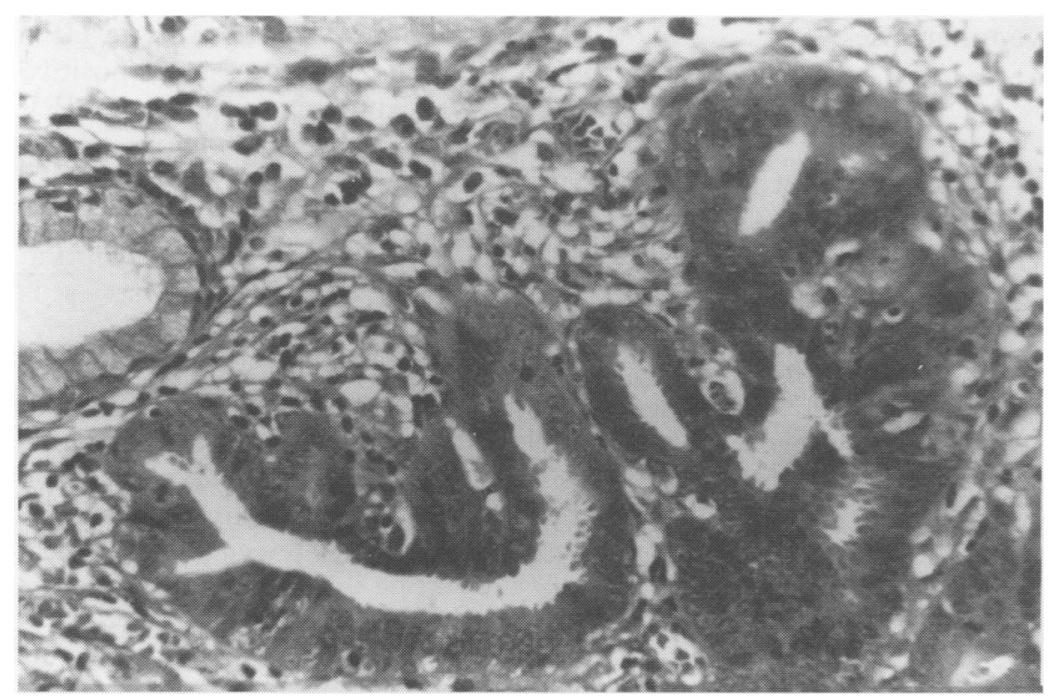

Figure 2 Adenocarcinoma in situ. There is nuclear enlargement and hyperchromasia. Nuclear multilayering involves up to full thickness of the lining epithelium. Mitoses are frequent.

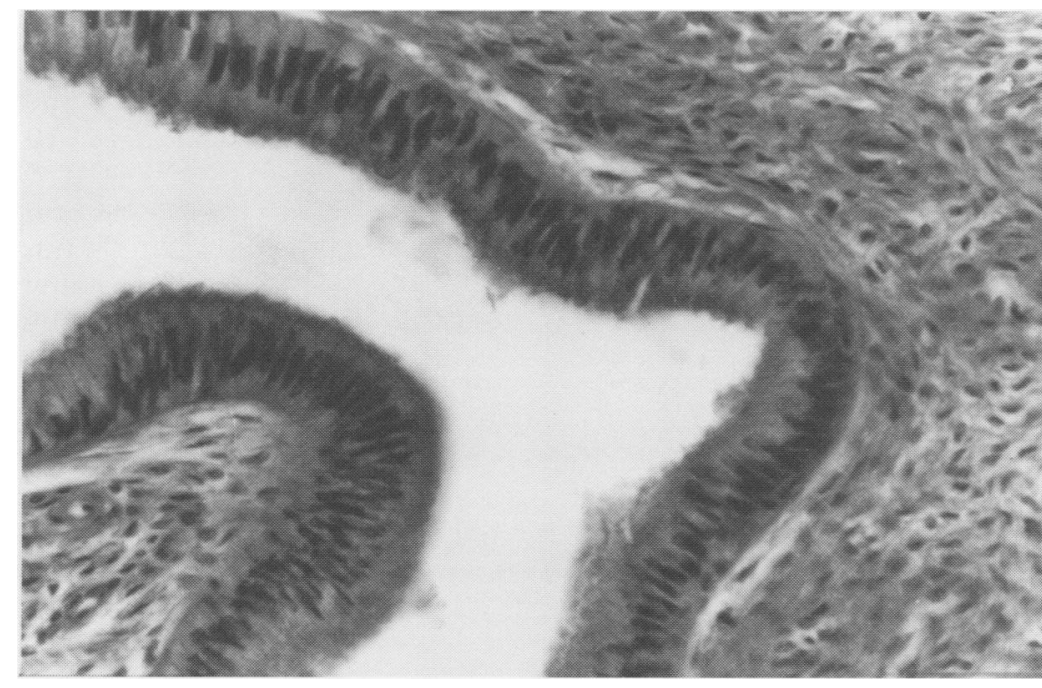

Figure 3 Glandular dysplasia of the uterine cervix. There is slight to moderate loss of mucin with less than two layers of nuclear stratification. Nuclei show low grade hyperchromasia and pleomorphism.
The morphological criteria used for diagnosis of adenocarcinoma in situ followed standard criteria ${ }^{11}$ and included nuclear enlargement and pseudostratification, involving up to full thickness of epithelium, nuclear hyperchromasia, and pleomorphism. There were also easily found mitotic figures and apoptotic bodies in these lesions. The amount of intracytoplasmic mucin in the affected glands was variably diminished. The nuclear chromatin was finely to moderately granular and evenly distributed. Nucleoli tended to be inconspicuous. Affected glands showed bud-like projections of glands into stroma and sometimes papillae (fig 1).

A sharp transition between normal and neoplastic epithelium was often seen (fig 2). A stromal reaction or strongly glandular complexity indicating invasion was absent in these adenocarcinoma in situ lesions. Histological subtyping of these cases was not carried out for the purpose of this study.

The cases considered to be endocervical glandular dysplasia showed lesser degrees of cytological atypia than AIS of the cervix. The morphological criteria followed those described by others for endocervical glandular dysplasia. ${ }^{512}$ There was no attempt, however, to divide cases into low and high grade dysplasia. The criteria used for diagnosis included slight to moderate loss of mucin in endocervical columnar cells showing nuclear irregularity, low grade nuclear hyperchromasia, and few mitotic figures. There were less than two layers of pseudostratification and glandular architecture was intact (fig 3). Care was taken to exclude cervical endometriosis and tubal metaplasia. In four cases of glandular epithelial multinucleation endocervical glands were seen to be focally lined by variable numbers of multinucleate giant cells. There was no nuclear atypia in these or adjacent cells (fig 4). Testing of the specimens for HPV was performed using a commercial kit, the ViraType in situ Human Papillomavirus Tissue Hybridization Kit (Life Technologies, Gaithersberg, Maryland, USA). This kit contains probes for three HPV groups HPV 6 and 11, HPV 16 and 18, and HPV 31, 33 and 35 . There is also a positive control probe (human genome DNA probe) and a negative control probe (PBR 322 DNA). Therefore, five sections were needed for each case.

Sections of a cervical biopsy specimen histologically diagnosed as showing koilocytotic atypia consistent with HPV infection and previously shown to contain HPV 16 or 18 were processed with the test specimens as a control for tissue processing and a control for all the kit reagents. Ten histologically normal cervical biopsy specimens were also tested as a control.

The protocol as set out in the ViraType kit instructions was followed with some minor modifications, as reported before. ${ }^{13}$

Testing was considered positive when the nuclei were stained a blue or purple colour. Immunoperoxidase stains for herpes virus was carried out on $5 \mu \mathrm{m}$ thick sections of two of the cases of endocervical epithelial giant 


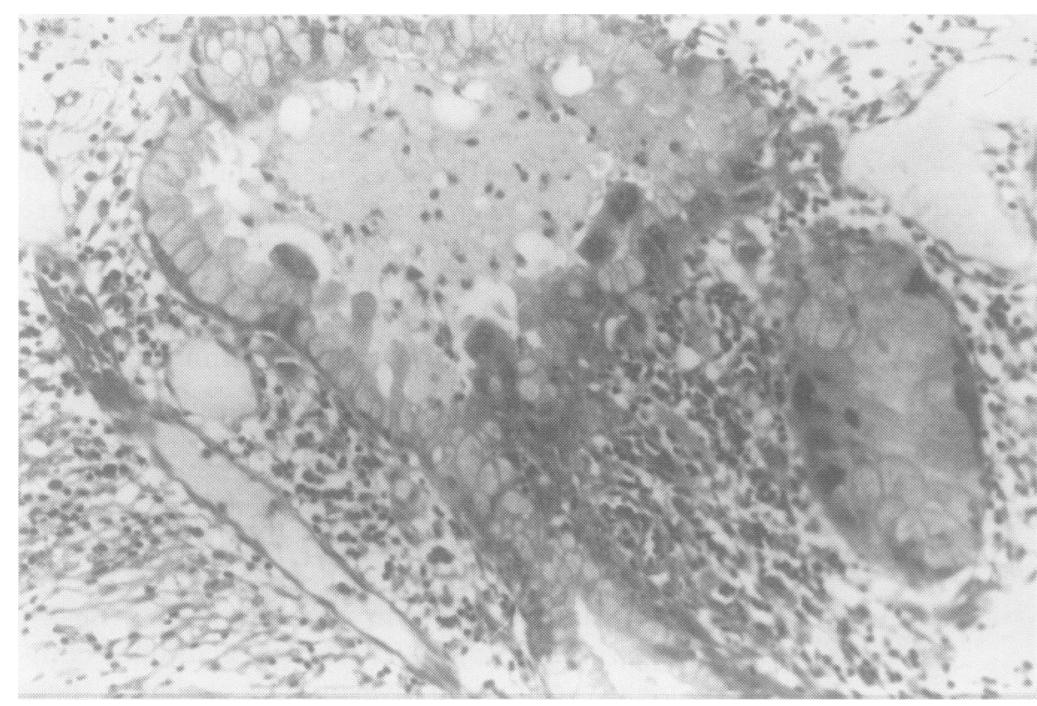

Figure 4 Multinucleate giant cells are seen lining the endocervical glands. The adjacent epithelium appears normal with small basal nuclei.

cell change. The focal nature of these lesions precluded study in the two other cases.

\section{Results}

Nine out of 21 cases of adenocarcinoma in situ tested positive with the HPV $16 / 18$ probe. Positivity was seen as a fine granular dark blue nuclear staining (fig 5) or dot-like nuclear staining in affected endocervical glands. One of the cases positive for HPV $16 / 18$ was also positive for HPV $31 / 33 / 35$. Three of four cases in which CIN was coexistent with adenocarcinoma in situ and present in the block selected for study, showed the presence of the same HPV type (16/18) within both the CIN and the adenocarcinoma in situ lesions.

None of the 10 cases of endocervical glandular dysplasia or the four cases of endocervical glandular epithelial multinucleation showed hybridisation with any of the HPV DNA probes used.

All 10 cases of normal cervical biopsy specimens tested were negative for HPV DNA. Immunoperoxidase stains for herpes virus infection was negative in the two cases of endocervical glandular epithelial multinucleation tested.

\section{Discussion}

Forty three per cent of adenocarcinoma in situ lesions in this study (nine out of 21 cases) contained HPV DNA in neoplastic cell nuclei using a DNA in situ hybridisation method with a commercial kit preparation. Tase et al examined 21 cases of adenocarcinoma in situ eleven of which also contained microinvasive adenocarcinoma, for HPV DNA by using ${ }^{3} \mathrm{H}$ labelled HPV 16 and HPV 18 probes $^{8} ; 70 \%$ of these cases were positive for HPV DNA. Using biotin labelled HPV DNA probes on paraffin wax sections with a streptavidin alkaline phosphatase detection system, Jaworski showed the presence of HPV DNA in $70 \%$ of cases. ${ }^{7}$ The variation in positive results in these different series is largely explained by the difference in sensitivity of the methods used. This was also found in another study using this methodology and which included some cases reported in this paper. ${ }^{14}$ Methods using radioactive isotope labelled probes are highly sensitive and detection may be possible with fewer than five viral

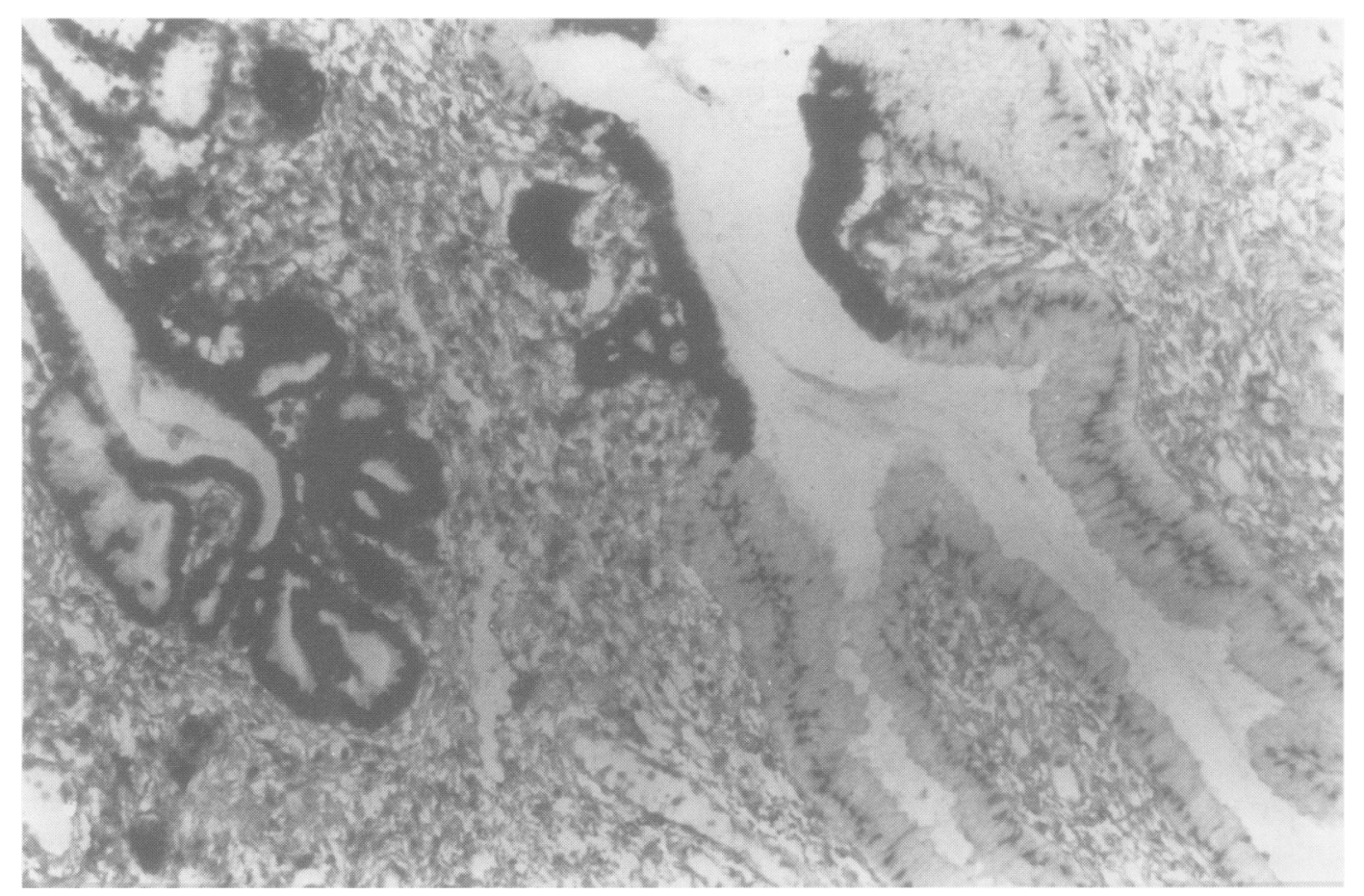

Figure 5 In situ hybridisation for HPV DNA 16/18. Dark blue granular nuclear staining detects presence of HPV within nuclei of this section showing adenocarcinoma in situ. Relevant unstained non-neoplastic nuclei can be seen (Methyl green counterstain). 
copies per nucleus. With the commercial kit preparations 10 to 20 viral copies are needed before HPV can be detected. A study using HPV mRNA expression showed the presence of HPV in 15 out of $17(88 \%)$ cases. $^{9}$ Geographic variation in prevalence of HPV may also account for part of the difference in results obtained from HPV studies of adenocarcinoma in situ and may explain why a recent study from the United Kingdom failed to reveal similar results. ${ }^{15}$

One of the cases of adenocarcinoma in situ positive for HPV 16/18 was also positive for $31 / 33 / 35$. This may be a case of mixed infection but this test kit uses lower stringency in order to achieve higher sensitivity and this is probably a more likely explanation.

None of the 10 cases of endocervical glandular dysplasia of the uterine cervix tested contained HPV DNA. If these lesions contained low viral copy numbers, detection may not have occurred using the commercial kit method. If these are pre-neoplastic HPV induced lesions, however, at least some cases should be detected by this method given the results obtained for AIS lesions. Alternatively, glandular dysplasia may not be lesion induced by HPV and hence not necessarily a preneoplastic lesion. These results support the findings by Tase et al and support the idea that many of these lesions of endocervical glandular dysplasia represent reactive changes. ${ }^{6}$

Microglandular endocervical hyperplasia has also been suggested as a possible HPV induced pre-neoplastic lesion. ${ }^{16} 17$ There does not, however, seem to be enough evidence for this. ${ }^{6}$

The cause of multinucleation of endocervical glandular epithelial cells remains obscure. No cytopathic changes or viral inclusions to suggest herpes virus infection are seen in these cases. Absence of herpes virus infection was confirmed in two cases by negative immunoperoxidase stains for herpes virus infection. It has been suggested that this multinucleation may be caused by HPV. ${ }^{5}$ None of the four cases of giant cell change tested for HPV, however, showed any positive reactions. If these are pre-neoplastic HPV induced glandular lesions comparable with pre-neoplastic HPV induced squamous epithelial lesions-namely, koilocytosisextremely high viral copy numbers should be present in their nuclei similar to the situation with koilocytes.

Therefore it seems highly unlikely that these lesions are induced by HPV. More sensitive in situ hybridisation studies have not been reported and ultrastructural studies are not available. Studies of this nature are warranted to clarify adequately the nature of these giant cells.

In conclusion, the results of this study show that while adenocarcinoma in situ is an HPV associated lesion, the virus probably plays little part in the development of endocervical glandular dysplasia or endocervical glandular epithelial giant cell change. We thank Mrs Lee-Ann Daley for typing the manuscript, for his advice and support, and, the laboratory staff of the Queensland Cytology Service for their technical assistance.

1 Reid R, Greenberg M, Jenson B, et al. Sexually transmitted papilloma virus infections. The anatomic distribution and pathologic grade of neoplastic lesions associated with different viral types. Am $\mathcal{F}$ Obstet Gynecol 1987;156:212-22.

2 Syrjanen K, Parkkinen S, Mantyjarvi R, et al. Human papilloma virus (HPV) type as an important determinant of the natural history of HPV infections in uterine cervix. Eur F Epidemiol 1985;3:180-7.

3 De Brux J, Ionesco M, Barrasso R. Papilloma virus infection and cervical intraepithelial neoplasia (CIN). Study of CIN III in 2 series of conization and hysterectomies. (1957-1968 and 1981-1983). Bull Cancer (Paris) 1985; 72:290-7.

4 Yun K, Molenaar AJ, Wilkins RJ. Detection of human papilloma virus DNA in cervical lesions by in-situ DNA hybridization. Pathology 1989;21:1-4

5 Brown LJR, Wells M. Cervical glandular atypia associated with squamous intraepithelial neoplasia. A premalignan lesion? ₹ Clin Pathol 1986;39:22-8.

6 Tase T, Okagaki T, Clark BA, Twiggs LB, Ostrow RS, Faras AJ. Human papilloma virus DNA in glandular dysplasia and microglandular hyperplasia: Presumed precursors of adenocarcinoma of the uterine cervix. Obstet Gynecol 1989;73:1005-8.

7 Jaworski RC. Endocervical glandular dysplasia, adenocarcinoma in situ and early invasive (microinvasive) adenocinoma in situ and early invasive (microinvasive) adenocarcinoma of the

8 Tase T, Okagaki T, Clark BA, Twiggs LB, Ostrow RS, Faras AJ. Human papilloma virus DNA in adenocarcinoma in situ, microinvasive adenocarcinoma of the uterine cervix, and co-existing cervical squamous intraepithelial neoplasia. Int f Gynecol Pathol 1989;8:8-17.

9 Farnsworth A, Laverty C, Stoler MH. Human papilloma virus Messenger RNA expression in adenocarcinoma in situ of the uterine cervix. Int $\mathcal{f}$ Gynecol Pathol 1989;8: 321-30.

10 Tase T, Sato S, Wada Y, Yajima A, Okagaki T. Prevalence of human papilloma virus type 18 DNA in adenocarcinoma and adenosquamous carcinoma of the uterine cervix occurring in Japan. Tohoku f Exp Med 1988;156:47-53.

11 Gloor E, Ruzicka J. Morphology of adenocarcinoma in situ of the uterine cervix. A study of 14 cases. Cancer 1982;49:294-302.

12 Gloor E, Hurlimann J. Cervical intraepithelial glandular neoplasia (Adenocarcinoma in situ and glandular dysplasia). A correlative study of 23 cases with histologic plapla). A corselogic grading, histochemical analysis of mucins and immunohistochemical determination of

13 Wright RG, Murphy DP, Gupta AC, Cox N, Cooke RA Comparative in situ hybridization study of juvenile laryngeal papillomatosis in Papua New Guinea and Australia. $尹$ Clin Pathol 1990;43:1023-5.

14 Nicklin JL, Wright RG, Bell JR, Samaratunga H, Cox NC, Ward BG. A clinicopathological study of adenocarcinoma in situ of the cervix. The influence of cervical HPV infection and other factors and the role of conservative surgery. Aust NZ $\mathcal{f}$ Obstet Gynaecol 1991;31: 179-83.

15 Young FI, Ward LM, Brown LJR. Absence of human papillomavirus in cervical adenocarcinoma determined by in situ hybridization. $f$ Clin Pathol 1991;44:340-1.

16 Czenobilsky K, Kessler I, Lancet M. Cervical adenocarcinoma in a woman taking oral contraceptives. Obstet Gynaecol 1974;43:517-21.

17 Dallenbach-Hellweg G. On the origin and histological structure of adenocarcinoma of the endocervix in women under 50 years of age. Pathol Res Pract 1984, 179:38-50. 